\title{
Intermédialités
}

Histoire et théorie des arts, des lettres et des techniques

Intermediality

History and Theory of the Arts, Literature and Technologies

\section{Intermédialité, cinéma, musique}

La symphonie-histoire d'Alfred Schnittke ["Raconter / Telling", no 2 automne 2003]

\section{Johanne Villeneuve}

Numéro 20, supplément, automne 2012, printemps 2013

traverser

crossing

URI : https://id.erudit.org/iderudit/1023525ar

DOI : https://doi.org/10.7202/1023525ar

Aller au sommaire du numéro

Éditeur(s)

Revue intermédialités (Presses de l’Université de Montréal)

ISSN

1705-8546 (imprimé)

1920-3136 (numérique)

Découvrir la revue

Citer cet article

Villeneuve, J. (2012). Intermédialité, cinéma, musique : la symphonie-histoire d'Alfred Schnittke ["Raconter / Telling", no 2 automne 2003]. Intermédialités /

Intermediality, (20), 55-72. https://doi.org/10.7202/1023525ar 


\title{
Intermédialité, cinéma, musique La symphonie-histoire d'Alfred Schnittke'
}

\author{
Johanne Villeneuve
}

À Samuel Torello

\section{LE CONCEPT D'INTERMÉDIALITÉ}

Cette étude d'une narrativité intermédiale dans la musique du compositeur russe Alfred Schnittke nécessite, dans un premier temps, une mise au point quant à l'usage qui sera fait ici du concept d'intermédialité. Davantage qu'une longue introduction, cette première partie fournira donc l'essentiel d'une réflexion personnelle sur ce nouveau paradigme, non sans faire sienne la proposition formulée par Jürgen E. Müller, selon laquelle «l'approche intermédiatique constitue [...] un axe de pertinence» au lieu d'un «système fermé»».

En premier lieu, de manière superficielle - ce qui n’est pas péjoratif en l'occurrence - l'intermédialité se laisse concevoir comme l'interaction entre différents médias. Le concept trouve alors sa pertinence dans la diversité actuelle des médias et l'extraordinaire expansion des moyens de communication contemporains. La croissance rapide du multimédia et de ses enjeux économiques, la problématique de l'adaptation cinématographique ou télévisuelle d'une littérature à succès, les croisements de plus en plus nombreux entre la presse écrite et les chaînes d’informations télévisées, l'expansion des images numériques et la nécessité de s'inscrire dans une économie des savoirs sont autant de phénomènes qui rendent nécessaire la réflexion sur le rapport qu'entretiennent entre eux les médias.

1. Cette recherche a été menée grâce à l'aide financière du FQRSC dans le cadre d'un projet portant sur «l'intermédialité de l'expérience».

2. Jürgen E. Müller, «L'intermédialité, une nouvelle approche interdisciplinaire: perspectives théoriques et pratiques à l'exemple de la vision de la télévision », CINéMAS, «Intermédialité et cinéma », vol. 10, nºs 2-3, Montréal, printemps 2000, p. 106. 
Mais pour peu qu'on y réfléchisse, on se rend compte que la perméabilité entre les différents médias censée caractériser notre époque est, en réalité, la caractéristique des médias eux-mêmes.

En étudiant de plus près l'émergence de certains médias, on constate qu'il faut du temps avant que ne se cristallise un médium, avant que celui-ci ne fasse «institution ». D'un point de vue plus sociologique, donc, l'intermédialité se laisserait concevoir comme une des conditions d'apparition d'un nouveau médium. C'est le cas, par exemple, du cinéma dont l'émergence traduit un amalgame entre la photographie, le spectacle fantasmagorique et le music-hall, lorsque certains des premiers appareils du cinéma ressemblaient à des phonographes. Toujours dans une perspective historique, force est de constater qu'en certains points du globe, il en fut de même lors de l'apparition de l'écriture laquelle n'est jamais à proprement parler une «apparition » tant son instauration est lente et qu'en entretenant un lien compétitif avec la parole, elle maintient en elle la «trace» de la voix, comme la présence problématique de ce qui est amené à s'absenter.

Cet usage contextualisé du concept d'intermédialité a l'avantage de faire valoir la matérialité des médias et de mettre l'accent sur la difficile délimitation des frontières qui les départagent. Il n'est pas de tâche plus malaisée, lorsqu'on s'apprête à expliquer ce qu'est l'intermédialité, que de distinguer entre le médium et le support, entre le média institutionnalisé et la médiation au sens large (médiation narrative au sens de Paul Ricœur ${ }^{3}$, médiation du langage, médiation au sens juridique). Car la question demeure: qu'entendons-nous par «médialité » lorsque, par le moyen d'un simple préfixe (inter), nous prétendons ouvrir tout un champ de la recherche?

Ne faut-il pas alors prendre le problème frontalement, en liant la matérialité des médiations humaines (les supports, mais aussi les dispositifs techniques) à l'idéal de médiation qui consiste à «vivre ensemble»? La médialité serait alors le propre de toute organisation politique, voire de toute collectivité humaine dès l'instant où celle-ci se manifeste à elle-même le désir de la communauté. L'intermédialité serait le rapport institué entre, d'une part, les matérialités par lesquelles la communauté s'interpelle elle-même, se construit, conçoit ses échanges; et d'autre part, sa visée proprement politique, son idéal de communauté. C'est ce que Jean-Louis Déotte 4 appelle «l'appareil » lorsqu'il nomme

3. Voir Paul Ricœur, Temps et récit I-II-III, Paris, Éditions du Seuil, 1983-1985.

4. Jean-Louis Déotte, L'époque de l'appareil perspectif, Paris, L'Harmattan, coll. «Esthétiques », 2001. 
le système de représentation d'une époque (à la limite le jeu entre la représentation esthétique et la représentation politique). Lappareil permet de lier à une époque donnée le politique, l'esthétique et la technique - lier, par exemple, Machiavel à Descartes et à la peinture depuis le quattrocento. Est de l'ordre de l'appareil, selon Déotte, «ce qui fait époque». Si le politique paraît mettre en scène des acteurs s'exposant sur la place publique en toute immédiateté, et si l'esthétique paraît effacer la technique qui la met en œuvre, une prise en compte de l'appareil permet de faire voir que toute manifestation esthétique et toute construction politique émergent des formes de médiation, qu'elles sont liées entre elles comme l'expérience l'est à la technique.

Résumons-nous jusqu'ici: l'intermédialité est un concept qui déborde l'idée selon laquelle il puisse y avoir interaction entre des médias donnés. Le concept peut en effet désigner des époques dont la caractéristique première est l'émergence ou l'apparition. Il vise tantôt une pluralité de médias institutionnellement reconnus, tantôt le mouvement même de cette reconnaissance au cour de laquelle vient à paraître l'hétérogénéité d’un nouveau médium. Tantôt l'intermédialité a pour corollaire une hésitation entre deux ou plusieurs technologies médiatiques, tantôt elle assure, en tant qu'appareil, la jointure entre le politique et l'esthétique, faisant voir ce qu'ont en commun la médiation au sens d'un «vivre ensemble» et la médiation au sens des techniques qui rendent possible l'expérience esthétique.

On le voit bien: dans des situations concrètes, la mise en application du concept ne pose pas les problèmes de sa définition. On aperçoit sans problème la part intermédiatique dans l'adaptation d'un roman à l'écran, dans la construction d'un site Internet à partir de textes et d'images, et même dans la double occurrence du visuel dans l'élaboration du Discours de la méthode et la naissance de la perspective albertienne. C'est cependant la définition (au sens de la délimitation) de la médialité elle-même qui manque. Dans la mesure, en effet, où notre expérience ne peut ni se transmettre, ni s'élaborer sans médiation, comment alors délimiter le mouvement lui-même par lequel s'organise toute relation? Notre expérience, en effet, ne s'élabore jamais à l'écart des autres, dans une sorte de bulle, sans altérité ni résistance.

Depuis les débuts du Centre de recherche sur l'intermédialité de Montréal (CRI), plusieurs de mes collègues l'ont souligné : d'un point de vue strictement théorique, le terme «intermédialité » peut devenir caduc en vertu de sa signification abyssale, car il désigne un «inter» de l'inter : la médialité ou la médiation désignant d'emblée la fonction d'intermédiaire, l'intermédialité toucherait en quelque sorte la médiation des médiations. Cette différence de degré n’est 
cependant pas inintéressante. Elle rend sensible le fait que la modernité, à l'apogée de la culture visuelle, mesure la distance qui la sépare de la médiation traditionnelle fondée sur l'oralité. C'est en grande partie à cette mise en abyme que j’ai consacré ma communication sur Level Five (1996) de Chris Marker et la question de la mélancolie lors du premier colloque du CRI. Si la culture visuelle permet cette distanciation, la mesure des écarts et des délimitations, elle ouvre, avec la modernité, l'espace du paradoxe et du scandales.

Pour la culture orale traditionnelle, le «médium » est un milieu dans lequel on baigne. L'accent n'est pas mis sur ce qui est «séparé» et que le médium doit réunir, mais sur la processualité de la médiation en tant que performance. Technè et poièsis (la technique et le faire) ne font qu'un. La technique n'est jamais mise au service de l'art selon une conception instrumentale; il n'y a pas même de conception du tout. L'art et l'expérience sont enchevêtrés parce que la vie pratique détermine toutes les expressions, celles des humains comme celles des dieux. Pour la culture traditionnelle, il n'y a pas de séparation théorique entre la médiation et l'expérience, car la médiation est un milieu de vie et ne saurait être appréhendée autrement; on ne peut la rendre objective. Certes, on y assure des passages: quand le chaman traverse du côté des morts en chantant «avec la voix des anciens », il devient passeur, médiateur, mais il ne transgresse rien et ne provoque aucun scandale. La prise en compte de la fonction sociale du médiateur n'exige en rien que l'on distingue entre le sujet de la médiation et son objet. Comme la fluidité de la voix qui le guide, dans l'avènement de cette voix qui n'est plus tout à fait la sienne, le chaman se coule dans le milieu où les anciens rencontrent les vivants. La performance réactive alors la voix des anciens comme ce qui est toujours déjà là, dans une sorte de latence collective; toute séparation (d'avec les morts, les absents, les voyageurs) est ainsi vécue au quotidien, parce que le legs des anciens pèse toujours de son poids; les dieux sont toujours présents, tandis que le passé a la consistance du présent. Nul doute que les cultures traditionnelles sachent faire la part entre le vivant et le mort, mais comme il en est d'une eau qui coule d'une rive à l'autre, le médiateur effectue le passage en suivant le mouvement, parfois tranquille, parfois impétueux, qui le mène du monde des morts au monde des vivants, sans que l'on puisse établir précisément à partir de quel point-limite les morts cessent de guider les vivants.

5. Voir Johanne Villeneuve, «L'ordinateur de Chris Marker. Mélancolie et intermédialité », Protée, «Mélancolie entre les arts ", vol. 28, n 3 , Chicoutimi, hiver 2000-2001, p. 7-12. 
Cette lecture de la médiation comme milieu, je la dois à Walter J. Ong, mais aussi, indirectement, à Émile Durkheim et à Maurice Halbwachs ${ }^{6}$. Je rappelle brièvement l'idée durkheimienne selon laquelle les premiers hommes, loin de créer les dieux pour s'expliquer les phénomènes naturels qui leur auraient échappé sous la forme d'un mystère, les ont plutôt vus dans l'organisation sociale qui rattache les humains, comme la nature elle-même qui témoigne de relations entre les choses. Les divinités peuplent le monde à la manière des relations qui soudent la société elle-même, comme la nature elle-même: les arbres, les cours d'eau, la faune, les astres présentent un seul tissu organique où viennent éclore les divinités et se mirer les rapports entre les humains. Or, la modernité, si elle n'échappe pas aux conditionnements du milieu, à l'organisation sociale, induit une valeur supplémentaire. Elle s'arrache à la tradition orale de deux manières radicales: premièrement, en découlant d'une pensée logique fondée sur la technique de l'écriture pour laquelle le signe induit une conception de la médiation fondée sur la rupture d'avec le milieu, sur la distance, la réflexivité, l'objectivation et la conceptualisation - la médiation, qui perd alors de sa fluidité, produit des «documents », soit des objets dénués de « présence $^{7}$ »; deuxièmement, en se pliant au mouvement de la reproduction mécanique, ce qui implique une autre rupture, plus grande et démultipliée, soit des effets de vitesse rattachés aux médiations et qui incitent à penser que plus le médium est rapide, plus il est efficace, eût-il à s'effacer entièrement à force de vélocité.

Cette double incidence - contradictoire s'il en est - d'une distanciation réflexive à l'égard du médium et de l'effacement apparent de celui-ci, est justement ce que j’appellerais «l'intermédialité », dans son sens paroxystique, hautement problématique. En d'autres termes, l'intermédialité ne pourrait être thématisée que dans le contexte de la modernité. Elle devient ici le locus ou le moment de figuration du paradoxe - moment de la duplication à l'infini qu'impose l'idée d'une médiation qui est à la fois «lien» et « rupture », «efficacité » et « disparition ».

6. Voir Walter J. Ong, Orality and Literacy. The Technologizing of the World, Londres, New York, Routledge Press, 1982; Émile Durkheim, Les formes élémentaires de la vie religieuse, Paris, Librairie générale française, coll. "Livre de Poche », 1991 [1912]; Maurice Halbwachs, Les cadres sociaux de la mémoire, Paris, Albin Michel, 1994 [1925] et La mémoire collective, Paris, Albin Michel, 1997 [1950].

7. Sur l'extériorité des signes associée à l'invention de l'écriture, voir la lecture que fait Giorgio Agamben des Immémoriaux de Victor Ségalen: «L'origine et l'oubli. Parole du mythe et parole de la littérature», Image et mémoire, Paris, Éditions Hoebeke, coll. «Arts \& esthétiques», 1998, p. 45-63. 
La question que je me pose aujourd'hui est de savoir si on peut envisager les choses autrement, sans doute en d'autres contextes que la modernité, mais aussi dans le contexte de notre contemporanéité, en insistant moins sur le caractère abyssal du concept que sur la possibilité qu'il offre de penser les médias de manière anachronique. Car c'est sur le plan de la chronicité des médias, voire sur le plan d'une histoire des médias que l'intermédialité se révèle paradoxale, soit dans le passage d'une hégémonie médiatique à une autre (de l'oralité à l'écriture, à la culture des images, etc.) ; c'est dans la mesure où une histoire des médias prend forme que le médium s'ouvre à un champ de réflexivité qui lui permette de s'auto-appréhender en tant que médium, distinct des autres médias, quoique toujours en dette par rapport à ce qui le précède.

J'aimerais maintenant renverser cette logique en proposant une conception fondamentalement anachronique de l'intermédialité; non pas pour nier l'historicité des médias - ce qui invaliderait du coup la temporalité des rapports observés; ni davantage pour nier que certains médias font époque. Simplement pour montrer qu'en deçà des interactions entre les médias, des configurations institutionnelles et des prétendues hégémonies, préexiste quelque chose que j'aimerais appeler des «qualités médiatiques». Ces qualités, elles-mêmes historiquement chargées, fonctionnent plutôt comme des pôles de résistance ou de rupture à l'intérieur du médium; elles ne sont pas ce qui distingue un médium d'un autre ou le délimite, mais ce qu'un médium partage nécessairement avec d'autres matérialités que la sienne. Au caractère abyssal et problématique de l'intermédialité, je substitue les qualités d'une épaisseur des médias, celles d'une sédimentation où puisse encore se vérifier l'importance de la matérialité que suppose toute médiation. Il arrive, par exemple, que l'écriture emprunte à la voix la qualité de sa fluidité, ou que la voix œuvre à partir de l'écriture, comme sur un palimpseste, se fixant des normes qui sont celles de l'écrit et se figeant à l'oreille de celui qui écoute, comme les signes arrêtés sur les pages d'un livre; il arrive encore que le son puisse faire image, se laisser voir ${ }^{8}$. C'est, par exemple, l'image cinématographique de Pierre Perrault qui, dans Cornouailles (1994), fait ellemême trace en suivant le parcours des bêtes et les figures inscrites dans la roche. C'est encore l'écriture idéographique qui, aux dires d'Anne-Marie Christin", présuppose une pensée de «l'écran »; c'est l'image cinématographique qui fonctionne, comme la musique, sous la modalité de l'évanescence. L'intermédialité

8. Voir Gilles Deleuze, Cinéma 2. L’image-temps, Paris, Éditions de Minuit, 1985.

9. Anne-Marie Christin, L’image écrite ou la déraison graphique, Paris, Éditions Flammarion, 1995 . 
désigne alors un mélange de qualités médiatiques, mélange caractéristique de toutes les situations de médiation, puisque même l'oralité exige la double matérialité de la voix et du geste. Si tant est qu'il n'y a pas de médium premier, mais un contexte premier, soit l'oralité. Ce contexte fait appel à des qualités qui, si elles ont tendance à se cristalliser dans un médium précis selon les pratiques les plus courantes (la fluidité associée au son, la qualité d'archive associée à l'écriture), ouvrent à l'intérieur de chaque médium l'espace de l'intermédialité. Les qualités médiatiques ne trouvent pas leur origine à l'intérieur d'une seule pratique médiatique; c'est, au contraire, leur mélange qui permet la possibilité et la diversité des médiations. Les mélanges ne sont pas aléatoires et dépendent de conditions matérielles et de contextes culturels qui, eux, sont précis : l'homme préhistorique n'a pas inventé l'ordinateur, mais il a néanmoins trouvé, dans le ciel étoilé comme sur le chemin de la chasse, les qualités écraniques qui le portent au-delà de la seule sphère de l'immédiateté.

C’est sur la base de ces qualités médiatiques et de ce mélange que j’aimerais maintenant aborder la question de la narrativité dans une œuvre musicale d'Alfred Schnittke.

\section{LES ARCHIVES CINÉMATOGRAPHIQUES D’ALFED SCHNITTKE}

Le rapport qu'entretenait avec la musique le compositeur soviétique Alfred Schnittke était double: d'une part, l'œuvre dite «sérieuse » a eu une réputation internationale qui lui a valu de s'installer à Vienne où il a terminé sa carrière; d'autre part, le travail de Schnittke à titre de «compositeur de musique de films » dont il a su tirer expérience - la valeur artistique des nombreuses productions auxquelles il a participé étant toutefois assez inégale. L'intérêt de ce double emploi réside dans le fait qu'une très grande habitude à composer pour le cinéma a eu ses effets sur l'ensemble du travail de composition, y compris sur les œuvres réservées au répertoire des concerts. Je retiendrai deux effets importants qui ne sont pas étrangers l'un à l'autre : le sens de l'intrigue qui pénètre ces compositions musicales, et la dimension archivistique qui, de la matérialité des films en tant que «documents de travail », passe dans la musicalité.

En 1974, Schnittke achève la composition d'une œuvre pour orchestre, soit la Symphonie $n^{\circ} 1^{10}$. Dans sa préface, le compositeur s'explique sur ses motivations:

10. On trouvera l'enregistrement de la Symphonie $n^{\circ} 1$ d'Alfred Schnittke par l'Orchestre du ministère de la culture d'U.R.S.S. (dirigé par Gennadi Rozhdestvensky) sur la vieille étiquette «Melodia». 
Tout en composant la symphonie, depuis quatre ans je travaillais simultanément à la musique du film de Mikhaïl Romm Je crois [I vsyo-taki ya veryu, 1974]. En compagnie de l'équipe de réalisation, j'ai visionné des milliers de mètres de films documentaires. Petit à petit, ceux-ci ont formé dans mon esprit une chronique du $\mathrm{Xx}^{\mathrm{e}}$ siècle, apparemment chaotique, mais intérieurement ordonnée $\mathrm{e}^{11}$.

Dans son appréciation stylistique de l'œuvre, le chef d'orchestre Rozhdestvensky insiste sur deux traits particuliers: le procédé de «téléscopage-microscopage » et son caractère de «fabrication », plus précisément en russe sa "mémoire de fabrication $^{12}$ » (factura ayant ici le sens de «facture» et de «mémoire»). Peu s'en faut pour conclure au postmodernisme de Schnittke dont la symphonie exhiberait un caractère volontairement factice; à la première écoute, on ne peut qu'être frappé par l'intertexte et ses effets de parodie où se rejoignent la Cinquième symphonie de Beethoven, Stravinsky, le jazz et la musique militaire. Les procédés que sont l'emprunt (ou la « reprise » de motifs connus) et la citation dévoyée décrivent sans doute bien le travail du compositeur qui, non sans créer de polémiques, contraste avec le courant le plus important de la musique contemporaine. Cependant, la thèse «postmoderniste» voile une vérité plus profonde de l'œuvre, comme si l'architectonique audacieuse et spectaculaire de la symphonie voilait elle aussi la complexité d'une archéologie: c'est un travail de stratification qui m’intéresse ici, parce que c'est de lui que découlent les qualités médiatiques dont je viens d'énoncer l'importance à l'égard du concept d'intermédialité.

Si Rozhdestvensky, bien qu'occupé à décrire la stylistique musicale de la symphonie, s'arrête sur la factura de la fabrication et sur le procédé de «téléscopage-microscopage », c'est justement parce qu'on y reconnaît un travail de mémoire foncièrement intermédial qui, par couches successives, conduit au devenir anachronique de l'œuvre. De larges pans sonores avancent et se retirent, se cristallisent et se désintègrent, ponctués de petites scènes absorbées à leur tour par les couches surdimensionnées du temps. Or tout ce travail s'organise autour de deux axes narratifs: une impossible histoire de la musique en miroir d'une impossible histoire humaine; une mise en intrigue intermédiatique et souterraine dont le pôle d'attraction est l'indescriptible événementialité de la Seconde Guerre mondiale.

La symphonie est composée de quatre mouvements, Moderato, Allegretto, Lento et Lento Allegro. L'auditeur y retrouve une figuration liminaire du temps, les péripéties d'une musique en train de se consumer, des effets narratifs

11. Alfred Schnittke, Symphonie n ${ }^{\circ}$ 1, Étiquette «Melodia» (livret). Je traduis.

12. Symphonie $n^{\circ} 1$ (livret). 
d'anticipation et des plages de réminiscences qui remontent le temps avant de le suspendre et de l'annihiler. Plus sensiblement dans le Lento, la suspension crée une tension narrative, comme si la musique se jaugeait et redoutait sa propre précipitation, mettant en valeur la disparité d'images sonores et la difficulté du mouvement; après un changement brutal de séquence, le dernier mouvement introduit une assomption bouleversante, faisant large place à l'autorité narrative qui, par une intervention catastrophiste puis apaisante, aspire dans le silence la force auparavant déployée, attendue et étendue par la performance de l'orchestre.

Si j’use des termes «autorité narrative», «séquence», «images sonores", «tension narrative», ce n'est pas tant pour reconnaître à l'ontologie même de la musique la composante de la narrativité, mais parce que la Symphonie $n^{\circ} 1$ ne peut être saisie dans sa dimension archéologique sans égard pour ses qualités cinématographiques qui passent, chez Schnittke, par une habitude «musicale» des intrigues filmiques. Rappelons-le: Schnittke a passé sa vie à écrire la musique de films fortement narratifs, tandis que la Symphonie $n^{\circ} 1$ est issue d'une expérience singulière de visionnage cinématographique. Mais contrairement à ce que l'on pourrait croire, la grande force «cinématographique » de la symphonie ne repose pas sur la qualité même du «montage ${ }^{13}$ » - bien qu'on puisse aussi parler d'un effet de «mixage» - , mais sur une «mise en scène » du montage/ mixage en tant que "performance». C'est cette mise en scène qui participe du sens de l'intrigue. Car les images sonores qui traversent la symphonie ne sont jamais que les réminiscences de ce qui est déjà sur la table de montage: les images du siècle visionnées par le compositeur, lequel, pour l'occasion, occupe la fonction d'un chroniqueur ${ }^{14}$. Devant lui défilent des images enregistrées, tantôt

13. À l'inverse de l'usage de la notion de «montage» en musique, on trouvera chez Sergei Eisenstein toute une isotopie de «l'orchestique» pour décrire le travail du montage cinématographique. Ainsi, dans un texte datant de 1938 (voir Le film, sa forme, son sens, trad. Armand Panigel, Paris, Christian Bourgois, 1976, p. 256-264) où il expose sa théorie du «montage vertical », il parle de «polyphonie», puis décrit le mouvement d'un orchestre qui progresse horizontalement.

14. Ces «images sonores» peuvent être aussi bien les réminiscences d'images visuelles appartenant à des films d'archives sans son que les souvenirs d'images-sons. Dans ce dernier cas, l'image visuelle devient inséparable de l'image sonore, dans la mesure où le visionnage d'un film sonore n'implique pas de séparation entre la part visuelle du médium et sa part sonore. Je partage à cet égard les propos de Michel Chion qui s'insurge contre l'usage de l'expression «bande-son »: «Lorsqu'il y a, dans n'importe quel film, un certain nombre de couches sonores simultanées mises sur une image, sur une scène, ces sons sont loin de se constituer en troupe, en bande, avant d'être confrontés à l'image, mais chacun d'eux, selon le rapport particulier et momentané qu'il a avec l'image, est réparti, 
sans le son, tantôt accompagnées d'un son lui-même souvent remixé, mais néanmoins «empreinte[s] unique[s] des lieux qui ont été investis ${ }^{15}$ ». La symphonie a donc pour point de départ une double occurrence du médium cinéma: une habitude narrative dont le sens de l'intrigue prévaut en relation avec le mouvement enregistré de l'image, qui cadre (microscopie, téléscopie) et divise l'espace sonore en plans, en séquences, en champs, hors-champs ${ }^{16}$ et contre-champs; puis une expérience de l'archive cinématographique qui fait apparaître, en creux, à la fois l'absence chaotique de montage et l'ordonnance souterraine, voire secrète, d'une histoire anachronique.

\section{L'ENCHANTEMENT INTERMÉDIATIQUE ET LA NARRATIVITÉ}

64 Dans un article proposant la synthèse des théories sur la narrativité de la musique au cinéma, Jerrold Levinson établit une distinction entre l'auteur du film (film maker) et le narrateur cinématique, entre la fabrication du film et la production d'une vérité fictionnelle ou, pour le dire autrement, entre la construction du film et la conduite du récit ${ }^{17}$. Dans cette optique, Levinson se demande si la musique opère une transformation fictionnelle ou non fictionnelle dans un film: «Who or what is responsible for such music ${ }^{18}$ ? » demande-t-il, associant immédiatement le critère de fictionalité à la valeur narrative de la musique. Or, tout récit n'est pas «fiction » et toute fiction n’engage pas nécessairement une narration. La question que pose Levinson rappelle celle que posait Wolfgang Kayser en 1958 dans un texte célèbre intitulé, justement, "Qui raconte le roman" ? ». La narrativité y est

situé, interprété, soit dans l'image, soit dans ses coulisses, soit dans un autre lieu invisible, dans un futur (voix de narration) ou dans une fosse d'orchestre imaginaire.» (Michel Chion, «Les chiens de faïence », Protée, «Sons et narrations au cinéma », Chicoutimi, vol. $13, \mathrm{n}^{\circ} 2$, été $1985, \mathrm{p} .7$ )

15. Je paraphrase ici le texte très riche que proposent, au sujet du son au cinéma, Serge Cardinal, Martin Allard et Louis Comtois, «La musicalité d'une bande sonore. À propos de L'invention d'un paysage», dans Réal Larochelle (dir.), Écouter le cinéma, Montréal, Éditions Les 400 coups, coll. «Cinéma », 2002, p. 158-178.

16. Au concept visuel de «hors-champ» s'ajoute le concept sonore de «son off» (ou «voix off»).

17. Voir Jerrold Levinson, «Film Music and Narrative Agency», dans David Bordwell et Noel Caroll (dir.), Post-Theory: Reconstructing Film Studies, Madison, University of Wisconsin Press, 1996, p. 248-282.

18. Jerrold Levinson, «Film Music and Narrative Agency», p. 248.

19. Wolfgang Kayser, «Qui raconte le roman? », dans Roland Barthes et al., Poétique du récit, Paris, Éditions du Seuil, 1977, p. 59-84. 
associée à la faculté de «mentir ». Parce que l'auteur ne saurait «mentir », suggère Kayser, il doit se métamorphoser en narrateur, comme le font les parents qui se métamorphosent devant leurs enfants pour leur raconter des histoires. «Dans l'art du récit, le narrateur n'est jamais l'auteur, déjà connu ou encore inconnu, mais un rôle inventé et adopté par l'auteur ${ }^{20}$ ». Le narrateur «ne raconte pas par la grâce d'une bonne mémoire, il voit le passé comme présent grâce à une faculté plus qu'humaine ${ }^{21} »$.

Les attributs de la musique de film sont donc, pour Levinson, analogues à ceux du narrateur tels que décrits par Kayser: le narrateur « dispose de pouvoirs réservés aux dieux» sans commune mesure avec des «situations terrestres ${ }^{22}$ ». Lopposition sous-entendue entre un pouvoir narratif analogue au pouvoir divin et une mémoire terrestre, de même que la nécessité de séparer l'auctorialité poïétique d'une propension narrative au mensonge attestent le lieu commun de la modernité selon lequel la proximité du divin par le mythe ouvre la possibilité du mensonge. C'est bien ce lieu commun qui constitue l'arrière-fond de la narratologie filmique lorsqu'elle suppose qu’en «faisant avancer le récit », la musique de film appartient nécessairement à la sphère de la fiction.

À l'encontre du critère de «fictionalité », la narrativité de la musique peut être comprise dans les termes plus neutres d'une mise en intrigue qui, au sens de la narration traditionnelle, cause un enchantement sans conduire au mensonge: le muthos ne dépend pas d'une «séparation » de la sphère de la réalité, il consiste au contraire à « prendre ensemble» un divers d'incidents ${ }^{23}$. Cette conception du récit n'est pas étrangère au fait que, dans ses manifestations premières, la narrativité est entièrement liée aux modalités de l'oralité et du chant: contrairement à la «vision ", capable de disséquer et de discriminer jusqu'à refuser ce qui se présente à elle - en fermant les paupières, par exemple —, le son «incorpore».

20. Wolfgang Kayser, "Qui raconte le roman?», p. 71.

21. Wolfgang Kayser, "Qui raconte le roman?», p. 74. Kayser écarte systématiquement la portée de Mnemosyne dans la sphère de la narration, se contentant de nier «l'importance d'une bonne mémoire».

22. Wolfgang Kayser, "Qui raconte le roman?», p. 79.

23. L'expression qu'emploie Aristote dans la Poétique est: è tôn pragmatôn sustasis, que certaines traductions donnent injustement comme «agencement des faits en système» (voir la critique qu'en fait Paul Ricœur, Temps et récit I, p. 57). La traduction de Hardy donne «assemblage des actions accomplies» (Aristote, Poétique, trad. J. Hardy, Paris, Éditions Les Belles Lettres, coll. «Budé», 1932, p. 37). «Sustasis» ne peut renvoyer à un «système» (anachronique), mais littéralement à «ce qui se tient ensemble », d'où l'idée que le muthos consiste à «prendre ensemble » un divers d'incidents, de l'hétérogène. 
«The auditory ideal, by contrast, is harmony, a putting together ${ }^{24}$ », écrit Walter J. Ong. On sait, bien sûr, que la poétique aristotélicienne prend appui précisément sur la tradition orale dont elle décrit l’héritage épique jusque dans les tragédies. Il n'est donc pas étonnant qu'on y affirme que la fonction du récit - au sens de la mise en intrigue - consiste à «prendre ensemble » un divers. De part en part, la narrativité, dans sa version la plus ancienne, est déterminée par les qualités médiatiques du son : sa fluidité et son évanescence qui conditionnent la nécessité de la mémorisation; son incorporation qui lie toute manifestation linguistique à la performance du corps; son événementialité qui fait de toute parole une unité d'action et de toute manifestation du passé une manifestation dans le présent. Si la narration permet de «voir le passé comme présent », comme le pense Kayser, c'est parce qu'à l'origine même de la narrativité, l'événementialité du son, en tant que «présent», demeure paradoxalement inscrite dans d'autres médias comme le texte ou le film. La «faculté plus qu'humaine» invoquée par Kayser pour expliquer la situation du narrateur de roman est, en cela, tributaire des Muses. C'est pourquoi le rôle de la musique dans la narrativité filmique s'explique difficilement par le seul truchement de la catégorie de la «fiction »; la simple composante musicale d'une intrigue cinématographique suffit à rendre les échos lointains de cette présence des Muses - non pas la présence romantique du surnaturel, mais les traces tantôt discontinues, tantôt harmonieuses, de ce qui lie, dès l'origine, la narrativité au chant. Divinité, musique, désir terrestre et mémoire se conjoignent ainsi dans l'action des Muses, filles de Zeus et de Mnemosyne ${ }^{25}$.

Au sens de la culture orale antique, la «fabrication» narrative, pour reprendre les termes de tout à l'heure, est une poièsis dont on ne peut négliger le caractère technique et pratique. Lié à l'efficacité de l'acte en tant que «fabrication ", le technème de l'univers homérique trouve dans la fabrication des armes son exemple premier : «Déjà chez Homère, écrit Castoriadis, s'accomplit le passage de ce sens [la fabrication des armes] à celui de causer, faire être, amener à l'existence, souvent détaché de l'idée de fabrication matérielle, mais jamais de celle de l'acte approprié et efficace ${ }^{26}$. » Toutefois, ce que Castoriadis néglige ici, à travers l'idée d'un détachement de la fabrication matérielle, est que la matérialité

24. Walter J. Ong, Orality and Literacy, p. 72.

25. Sur les Muses en rapport à la poésie acoustique et la technique, voir le texte de Jed Rasula, "Poetry's Voice-Over», dans Adalaide Morris (dir.), Sound States: Innovative Poetics and Acoustical Technologies, Chapel Hill et Londres, The University of North Carolina Press, 1997, p. 274-317.

26. Cornelius Castoriadis, Les carrefours du labyrinthe 1, Paris, Éditions du Seuil, 1978 , p. 291. 
s'exprime de toute façon dans l'insistance que l'on met à faire voir le geste. Il n'y a pas d'artisanat sans implication totale de la matière dans le geste de la fabrication; simplement, il ne saurait y avoir «d'idée » de la fabrication matérielle, parce que la matière elle-même passe par le pneuma du vivant, par un souffle - condition première de l'oralité : les dieux y sont toujours présents à travers l'acte générateur de la création. Littéralement, la poésie est «ouvrage» inspiré par les Muses, et scelle l'épreuve d'un faire toujours ancré dans la vie pratique. On ne sera pas surpris de découvrir dans le bouclier d'Achille, au lieu de la «représentation » du monde - ce qui serait le signe d'une «extériorisation» possible du monde par rapport à l'acte de sa création — le «monde» lui-même :

Homère ne dépeint pas, ne reproduit pas, n’imite pas, ne représente pas. Le monde est comme il est, allant son chemin de monde. C'est grâce à la même médiation de l'artisan que le poète nous le rend sensible. Homère ne contemple pas directement.

Il regarde faire. La forme n’a pas éliminé la vie en la représentant ${ }^{27}$.

Cette persistance du vivant dans l'artefact ou l'arme, on la reconnaît dans la poésie orale parce que celle-ci est inséparable de l'acte performatif qui la réitère en même temps qu'il la met au monde, en une seule fabrique. Ici encore, le souffle de la divinité n'est pas un vent qui passe et laisse sur son passage quelques souvenirs; les Muses animent de la vie elle-même une parole immémoriale, et la mémoire commune que garantit cette parole dépend donc de la matérialité bien vivante du corps de l'aède. Dans la perspective de sa mort, l'aède doit transmettre cette mémoire à d'autres, mais c'est aux corps de ses auditeurs autant qu'au corps communal qu'il s'adresse. Pour la culture orale, la voix est toujours un corps : la performance est toujours une «structuration corporelle ${ }^{28}$ » en tant que présence.

Dans le contexte d'une telle imbrication du vivant et de «l'image» (si l'on reprend l'exemple du bouclier d'Achille), la narrativité, au sens de la poièsis, n'a rien à voir avec le critère beaucoup plus tardif de «fictionalité ». On comprendra toutefois que le caractère vivant de l'image, en tant que signe performatif d'une corporalité à l'ouvrage, dépend du caractère oral de la médiation narrative et des qualités médiatiques qui traversent cette dernière et la définissent: la fluidité et l'éphémérité du son, la force et la fragilité du geste, la présence et l'impétuosité d'un corps, le caractère impératif de la performance et, finalement, sur le modèle

27. Jackie Pigeaud, «La création du monde ou le bouclier d'Achille», dans L'art et le vivant, Paris, Éditions Gallimard, coll. «NRF essais», 1995, p. 23.

28. Voir Paul Zumthor, «Le geste et la voix», Hors-Cadre 3, Paris, Centre National des Lettres, 1985 , p. 73-87. 
du son lui-même, l'absence de discrimination entre intériorité et extérioritée ${ }^{29}$. La culture orale induit une conception de l'expérience comme plénitude, densité, pour laquelle le principe de contradiction demeure inconnu; aussi les temps fusionnent-ils, de même que les espaces parcourus, dans l'unique creuset d'une collectivité en devenir de mémoire. Sur ce premier modèle, la médiation narrative, toujours empreinte de musicalité, a un fort pouvoir d'invocation; littéralement, elle fait être. La triple fonction d'incantation, de préservation et de transmission qu'occupe la poièsis à l'égard de la mémoire, se traduit par des opérations synthétiques - ce qu'Aristote retrouvait précisément dans la structure même de l'action représentée : la fusion de l'hétérogène est bien ce que permet le « poème », et plus explicitement encore, on s'en doute, quand celui-ci est chanté.

Dans un monde dominé par la prose, la narration devient un dérivé «pauvre» du chant, donc moins susceptible d'enchantements, traversé par des ordres de rupture, par des dénouements qui sont autant de déchirements de l'ethos ${ }^{3 \circ}$. Qu'en est-il alors de la narrativité filmique?

Une première hypothèse serait que celle-ci vient enrichir de nouveaux enchantements la narrativité moderne. Premièrement, parce que le médium filmique partage avec la musique certaines qualités qui échappent à l'écriture: la fluidité, les modes d'apparition et de disparition - et par conséquent l'impermanence d'une médiation toujours en devenir. Deuxièmement, parce que le cinéma a toujours entretenu avec le son une relation privilégiée, voire fusionnelle, soit dans le cinéma des premiers temps très fortement oralisé ${ }^{31}$, soit dans le cinéma dit «sonore» où les voix, les sons et la musique forment avec les images un seul et même alliage. Troisièmement, parce qu'en imprimant le mouvement sur la pellicule, le film opère à sa manière une fusion des temps: la «présence» du mouvement se confond avec le devenir de l'action et le «ça a été32» de la trace enregistrée. Cependant, contrairement à la poésie orale, le

29. Cette «liste» n'est, bien sûr, pas exhaustive mais seulement indicative. La compléter demanderait l'écriture d'un autre article.

30. Je me permets d'être très schématique dans le contexte de cet article. Pour un compte rendu plus détaillé de cette question, à savoir le devenir du sens de l'intrigue dans la modernité, voir mon livre Le sens de l'intrigue. La narrativité, le jeu et l'invention du diable, Presses de l'Université Laval, 2004.

31. Voir le très beau livre de Germain Lacasse à ce sujet: Le bonimenteur de vues animées. Le cinéma «muet» entre tradition et modernité, Québec, Paris, Éditions Nota bene, Méridiens Klincksieck, 2000.

32. On aura reconnu, bien sûr, la célèbre expression de Roland Barthes dans La chambre claire. Notes sur la photographie, Paris, Cahiers du Cinéma, 1980, p. 120-121. 
film demeure une archive bien matérielle; en sa qualité de «trace matérielle» indépendante du corps qui la produit et d'enregistrement, il participe de cette reproductibilité technique dont Walter Benjamin avait annoncé l'importante dissémination, et qui s'oppose radicalement aux médiations antérieures, tout en leur empruntant certaines qualités 33 .

Lobjet qui m’intéresse, à savoir le croisement de la narrativité musicale et de la narrativité au cinéma, engage donc deux trajectoires en apparence difficiles à concilier: la narrativité filmique en tant que dérivé pauvre, fragmentaire du chant, et la narrativité musicale en tant qu'opération synthétisante qui fonctionnerait telle une «mémoire en action » du corps performant. Comment l'une et l'autre, à l'intérieur d'un même processus médiatique (la Symphonie $n^{\circ} 1$ de Schnittke, par exemple), se rejoignent-elles?

Ma seconde hypothèse est que l'intermédialité se définit alors comme l'assomption de ce qui, très précisément, se creuse entre les deux médias que sont le cinéma et la musique, mais qui ressortit néanmoins aux «traces de l'oralité » en chacun d'eux. C'est lorsque le cinéma devient incantatoire et synthétique, plus près d'une mémoire du vivant, que la narrativité filmique devient en elle-même «musicale» - même sans trame musicale; en retour, c'est lorsque la musique, fortement pneumatique, se confond avec «l'action» tout en exhibant des ordres de rupture, du morcellement, de la fragmentation, toutes manifestations de la présence de «traces », que la narrativité musicale devient « cinématographique ». L'expression même «traces de l'oralité » est en soi litigieuse puisqu'elle confronte l'impermanence caractéristique de la médiation orale à la persistance matérielle des traces. Or, c'est bien ce litige que met en scène la symphonie de Schnittke, en donnant à la narrativité musicale la consonance obscure d'une mémoire documentaire, en instillant une force pneumatique à des échantillons enregistrés de l'histoire du Xx ${ }^{\mathrm{e}}$ siècle. En écoutant la symphonie, l'auditeur suit la trame synthétique d'une fusion mémorielle autant qu'il est troublé par le tourbillon des disparitions et l'impossibilité de retenir au-delà de l'oubli les vecteurs du temps historique.

Dans son étude, à l'instar d'autres théoriciens du cinéma et à la manière des narratologues de la première heure, Levinson découpe en cinq fonctions la narrativité de la musique au cinéma: (1) faire avancer le récit; (2) créer une atmosphère; (3) dessiner une continuité en arrière-plan; (4) maintenir la

33. D’où la difficulté que Benjamin a, dans son célèbre essai, d'éliminer complètement l'aura de la sphère de la reproductibilité technique, élément pourtant central dans la théorie qu'il expose alors. 
tension; (5) contribuer au sens de la clôture. Ce qui manque toutefois à cette classification, c'est une considération pour les traits de mémoire qui lient la musique, l'image cinématographique et la narrativité. J'irais plus loin encore: les cinq fonctions mentionnées par Levinson témoignent d'une lecture toute particulière de ce qui constitue une intrigue, proche de la poétique aristotélicienne revisitée par la modernité et vidée de ses enchantements, au profit d'une efficacité qui frôle l'instrumentalisation. La musique au cinéma sert, semblet-il, un but déterminé sur un autre plan du film. Ainsi, la narrativité est-elle abstraite du mouvement même qui la porte, comme on obtiendrait une essence du cours même de l'eau. Certes, la production de tensions, l'agencement du possible et de la clôture narrative, les effets de continuité et la pulsion qui porte le récit vers l'avant sont des traits narratifs reconnus depuis longtemps dans l'étude des récits ${ }^{34}$. Mais au-delà de ce que la musique contribue simplement à illustrer et à dramatiser, elle est elle-même la manifestation des Muses, à la source de la narrativité et indéfectiblement attachée à la mémoire. Par conséquent, si tant est qu'on refuse de la reléguer à sa fonction instrumentale, la musique a pour effet, au cinéma, de réveiller les forces enchanteresses de son passé et de faire oublier «ses instruments » à la faveur d'un effet de présence dont on retiendra surtout le trait fusionnel et l'anachronisme. C'est ainsi que la musique "parle » et contient des «visions », qu'elle incorpore la vie par son acte de présence, produisant à son tour du « corps».

Si l'on reprenait les choses à propos de la Symphonie $n^{\circ} 1$, il faudrait donc considérer ceci: passant de la musique au cinéma dans la musique-cinéma, Schnittke fait faire un tour de plus à l'écrou qui enserre la musique à la narrativité filmique. Rappelons qu'en procédant par «dérivations » d'images sonores, la symphonie reprend des motifs musicaux connus pour les faire glisser, voire déraper, sur des trames typiques de musique d'archives cinématographiques: marches militaires et fanfares, jazz, etc. C'est la valeur d'enregistrement de ces « archives » que l'on expose dans ce contexte, et non la valeur purement esthétique de la « reprise » ou de la «citation». Puis, par dérivation dramatique, la musique de Schnittke remonte aux souvenirs cristallisés, parfois pétrifiés, de formes dramatiques passées, dont l'opéra par exemple, en exploitant le rythme de l'action et des conflits: les instruments deviennent des protagonistes; différentes plages sonores entrent en lutte les unes avec les autres, à la manière des grandes dramatisations historiques du XIX ${ }^{\mathrm{e}}$ siècle, mais constamment menacées par leur propre

34. Voir en particulier l'ouvrage déterminant de Peter Brooks, Reading for the Plot, Oxford, Toronto, Oxford University Press, Clarendon Press, 1984. 
assourdissement. Il serait facile de voir dans cette mise en intrigue une simple ironisation, une «mise à distance » de l'histoire occidentale de la musique; ce n'est pas ce que propose Schnittke qui, fidèle à son emploi de compositeur de film, pénètre l'image au lieu de s'en affranchir, et révèle plutôt combien toute ironisation, par l'effet du temps, voit ses pointes s'éroder et se couler dans la densité infranchissable d'une mémoire musicale. Finalement, la symphonie fait dériver la poièsis elle-même, qui, prise dans le mélange des archives et des traces, acquiert momentanément un caractère fragmentaire et anachronique - chaque thème se résorbant par son extrême fragilité temporelle, chaque incantation étant comprise dans l'effet de sa rupture - , permettant à la symphonie de produire, à même son mouvement, des épiphanies, des «arrêts sur image » contenant leur propre impulsion de dissolution. Ainsi l'histoire du siècle apparaît-elle à Schnittke, sous la double modalité de l'assomption musicale et de la dissolution, de l'expressivité des images sonores et de leur assourdissement. Cette «chronique » est musicale et cinématographique parce que la mémoire musicale est, pour Schnittke, indissociable de la mémoire cinématographique du point de vue de l'expérience. Elle est synthétique (synthèse de l'hétérogène) et fragmentaire (archivistique), pauvre et saturée à la fois.

Or, ce qui procède, dans la symphonie, de l'enchantement pur tient à l'absorption commune des qualités cinématographiques et musicales au sein d'une performance qui met des corps en jeu. Contrairement à ce qu'on pourrait penser, le travail d'archives qui s'exprime dans la symphonie ne dépend pas lui-même de la technologie d'enregistrement; certes la symphonie rappelle de manière souvent mimétique les documents d'archives, mais à aucun moment, en concert, les musiciens ne quittent leurs instruments pour faire entendre des échantillons sonores pré-enregistrés. Les «archives» sont, au contraire, performées «live », chaque son entendu étant le produit d'un corps présent. Il y a donc «mise en scène» du montage/mixage en tant que «performance ». Le résultat est double: d'une part, l'orchestre dissout la valeur archivistique de l'enregistrement dans la performance en salle; d'autre part, et par la même occasion, les musiciens se trouvent à renverser l'opération mimétique traditionnelle qui consiste à «copier » l'original, en imitant à leur tour, par le biais d'une performance vivante, la reproductibilité technique. C'est alors la narrativité historique qui se trouve critiquée et renversée par cette transfiguration vivante de la technique. Le renversement anachronique n'équivaut pas pour autant à une surdétermination de l'acte de copier. Au contraire, la musique reflue vers sa source première qui est celle du corps présent de la performance orale, mais elle le fait cette fois en prenant à sa charge le lourd fardeau d'une mémoire en «restes ». 
Pour le spectateur dans la salle de concert, l'effet tient de l'enchantement, parce que les archives lui apparaissent bel et bien avec leur patine - mais la patine de ce qui justement n'en a pas. Il n'y a pas de «ça a été » qui puisse se manifester dans le cadre d'une telle performance, parce qu'il n'y a pas d'enregistrement; néanmoins, cela ne confère pas pour autant le statut de «fiction » à la musique de Schnittke. La raison en est complexe et nécessite de réitérer ce qu'il en est de la musique dès ses premiers enchantements. Si la patine de ce qui n'en a pas apparaît, c'est parce que le vivant s'exprime en elle à travers les corps des musiciens en acte, comme il s'exprimait autrefois dans la performance orale. L'enchantement - la patine de ce qui n'en a pas - n'a pas la consistance du factice mais celle du lien. Si la notion s'apparente à celle d'aura, on retiendra pour l'en distinguer le fait qu'en performant les archives, l'orchestre ne touche à aucune trace matérielle des archives elles-mêmes, mais s'enfonce, pour ainsi dire, dans les sources de ce qui s'en éloigne le plus : la culture orale et sa surdétermination de la présence. La matérialité de la trace se défait alors dans l'immatérialité immémoriale de la musique. Loin de la hantise et des spectres qui caractérisent l'univers auratique, l'enchantement participe du vivant.

Comment la patine de ce qui n'en a pas peut-elle donc apparaître? Sur le modèle de l'oralité, lorsque les conteurs font le récit du passé et se transmettent leur savoir de génération en génération, au fil d’une performance toujours renouvelée, il n'y a plus de traces purement matérielles de ce qui n'est plus, mais le lien vivant de ceux qui sont venus après. De la même manière, les qualités médiatiques, fortes du souvenir de la matérialité, irradient dans ce qui est et devient, en dépit de ce qui a été perdu. Loin de s'en tenir au jeu impuissant de l'ironisation, l'orchestre de Schnittke donne à voir et à entendre, selon la double formulation du geste et du son, une mémoire vivante, traversée par le flot des archives cinématographiques et des images sonores dont l'expérience contemporaine ne peut qu'être investie. L'enchantement est bien le comble d'une telle expérience, lorsque celle-ci rejoint, dans le vivant, la mémoire de la "présence » même: le chant. 